RESEARCH NOTE

\section{Molecular Epidemiology of HIV in Brazil: Polymorphism of the Antigenically Distinct HIV-1 B Subtype Strains}

\author{
MG Morgado/ ${ }^{+}$, ML Guimarães, \\ I Neves Jr, VG Veloso dos Santos*, \\ MI Linhares-de-Carvalho**, \\ LR Castello-Branco/**, FI Bastos***, \\ EA Castilho***/*****, B Galvão- \\ Castro****, V Bongertz, The Hospi- \\ tal Evandro Chagas AIDS Clinical \\ Research Group*/++
}

Departamento de Imunologia, Instituto Oswaldo Cruz, Av. Brasil 4365, 21045-900 Rio de Janeiro, RJ, Brasil

*Hospital Evandro Chagas, Instituto Oswaldo Cruz,

**Ambulatório da Previdência da Arquidiocese do Rio de Janeiro ***DIS-CICT, Fiocruz ****LASP/

CPqGM, Fiocruz $* * * * * C N-D S T$ e Aids, Ministério da Saúde, DF, Brasil

Key words: HIV-1 polymorphism - molecular epidemiology - HIV-1 B subtype

HIV is one of the most genetically polymorphic human emergent pathogens of this century. Two related viruses, HIV-1 and HIV-2 were identified as ethiological agents of the human acquired immunodeficiency syndrome (AIDS) and, while HIV-2 is mainly restricted to the African continent, HIV-1 is distributed worldwide (DJ Hu et al. 1996 JAMA 275: 210-216).

\footnotetext{
Supported by grants from the PNUD/National Coordination of STD and Aids/Brazilian Ministry of Health, GPA/WHO, Programa Institucional de AIDS/FIOCRUZ and Conselho Nacional de Desenvolvimento Científico e Tecnológico - CNPq.

${ }^{+}$Corresponding author. Fax: +55-21-280.1589. E-mail: morgado@gene.dbbm.fiocruz.br

${ }^{++}$The Hospital Evandro Chagas AIDS Clinical Research Group: B Grinsztejn, VC Rolla, MCG Gallhardo, MRC Guimarães and Frits Sutmoller

Received 11 March 1998

Accepted 16 April 1998
}

The global effort for HIV characterization has lead to the identification of different HIV-1 strains, which could be divided into groups $\mathrm{M}$ (majoritary) and $\mathrm{O}$ (outlier). The $\mathrm{O}$ group shows limited distribution to Africa (M Peeters et al. 1997 AIDS 11: 493-498) with sporadic cases described in Europe and in the United States (I Loussert-Ajaka et al. 1994 Lancet 343: 1393-1394, M Rayfield et al. 1996 MMWR 45: 561-564), and contains genetically and antigenically more divergent HIV-1 isolates. At least ten HIV-1 subtypes (A-J) belonging to the $M$ group have been identified worldwide so far, which diverge among themselves close to $30 \%$ and $15 \%$, respectively, in the env and gag regions (G Myers et al. 1996 Human Retroviruses and AIDS, Los Alamos National Laboratory, Los Alamos). Recombination intra and inter distinct HIV-1 subtypes seems to be highly frequent generating mosaic genomes, which increase virus diversity (E Sabino et al. 1994 J Virol 68: 63406346, DL Robertson et al. 1995 Nature 40: 249259). Indeed, based on the full length genome sequence, HIV-1 subtypes $\mathrm{E}$ and $\mathrm{G}$ are now recognized as mosaic genomes, with parts of the viral genome clustering with the A subtype and parts forming two clearly distinct clades designated respectively E or G [F McCutchan et al. 1996 AIDS 10 (Suppl 3): S13-S20]. Such diversity has potential implications for vaccine design, as well as for diagnostics, pathogenesis and epidemiology (Workshop Report from the European Commission and the Joint United Nations Programme on HIV/ AIDS 1997 AIDS 11: UNAIDS17-UNAIDS36).

The HIV pandemic is highly heterogeneous and composed of many epidemics with distinct local aspects, such as risk factors for transmission and subtype distribution, among others (Expert Group of the Joint United Nations Programme on HIV/ AIDS 1997 AIDS 11: UNAIDS 1-UNAIDS 15). At the end of 1997, 30.2 million people in the world were estimated to be living with AIDS; $90 \%$ in the developing countries. Brazil accounts for 120,399 AIDS cases registered until August 1997 (Brazilian Ministry of Health 1997 AIDS Epidemiological Bulletin, PN DST/AIDS September/ November 1997), and up to 500,000 individuals are estimated to be infected with HIV.

Several studies have been carried out in Brazil in the last five years in order to evaluate HIV diversity in our country. Taken together, the results showed the presence of $\mathrm{B}, \mathrm{F}$ and $\mathrm{C}$ HIV-1 subtypes (K Potts et al. 1993 AIDS 7: 1191-1197, M Morgado et al. 1994 AIDS Res Hum Retrov 10: 569-576, J Louwagie et al. 1994 AIDS Res Hum Retrov 10: 561-567, JC Couto-Fernandez et al. 1994 AIDS Res Hum Retrov 10: 1157-1163, WHO National Network for HIV Isolation and Charac- 
terization 1994 AIDS Res Hum Retrov 10: 13271343), as well as HIV-1 B/F recombinant viruses (Sabino et al. loc. cit., F Gao et al. 1996 J Virol 70: 1651-1667). Moreover, HIV-1 F subtype isolates from Brazil were shown to be quite divergent from those described in Romenia and Africa (C Bandea et al. 1996 Emerging Infect Dis 1: 91-93). More recently, HIV-1 D subtype was also identified in two cases of single (M Morgado et al. unpublished results) and dual (LM Janini et al. 1996 Virus Genes 13: 69-81) infections in Rio de Janeiro.

In spite of such diversity, B subtype viruses account for more than $80 \%$ of the HIV-1 infections in Brazil (B Galvão-Castro et al. 1995 Actualizaciones en SIDA 3: 173-178), at least in the southeast region, which accounts for $73 \%$ of the AIDS cases described so far. However, those studies have also shown that Brazilian subtype B isolates present two distinct amino acid sequence composition at the generally highly conserved crown of the gp120 V3 loop, suggesting that those isolates could also be split into two main groups, one corresponding to the USA/European B consensus sequence and a second one, called B", typically found in Brazil. Indeed, instead of the conserved "GPGR" sequence at the crown of the V3 loop, this variant has a "GWGR" motif associated with other amino acid substitutions in the adjacent regions leading to changes in the antigenicity and secondary structure of the corresponding protein (M Morgado et al. $1996 \mathrm{Mem}$ Inst Oswaldo Cruz 91: 339-342). As the V3 loop contains immunologically relevant epitopes for neutralizing antibodies and cell mediated immunity (H Takahashi et al. 1992 Science 255: 333-336, JN Billaud et al. 1994 Vaccine 12: 46-55), and positively charged amino acid associated to the virus biological behavior (RA Fouchier et al. 1992 J Virol 66: 3183-3187, D Bhattacharyya et al. 1996 AIDS Res Hum Retrov 12: 83-90), and is also important for susceptibility of HIV-1 to chemokines and second receptor usage (E Cocchi et al. 1996 Nature Med 270: 18111815), the differences observed in the B" variant may have potential influence on vaccine design and pathogenesis. Such data reinforce the importance of discriminating HIV-1 infection by these viruses in the molecular epidemiological studies conducted in Brazil.

Heteroduplex Mobility Assay was described as a very sensitive technique to define HIV-1 subtypes, facilitating large scale molecular epidemiological studies (E Delwart et al. 1993 Science 262: 1257-1261, MH Bachmann et al. 1994 AIDS Res Hum Retrov 10: 1345-1353). However, DNA sequencing has been used for the identification of the B" variant, which is a too expensive and time consuming technique. Serological studies using synthetic peptides containing the GWGR motif in the gp120 V3 loop were also able to discriminate these samples although there was some cross reactivity (CC Pau et al. 1994 AIDS Res Hum Retrov 10: 1369-1377, RM Hendry et al. 1996 Mem Inst Oswaldo Cruz 91: 347-348, V Bongertz et al. 1998 Mem Inst Oswaldo Cruz, this issue).

Based on the evaluation of the restriction map of the V3 region from DNA sequences corresponding to the B" variant, it was possible to identify the Fok I restriction enzyme which cuts the sequences $\operatorname{GGATG}(\mathrm{N})_{9}$ and $\operatorname{CCTAC}(\mathrm{N})_{13}$ present in the crown of the V3 loop coding the GW motif, capable of discriminating these samples from those corresponding to the conventional subtype B isolates. Indeed, this approach has been previously used by D Covas, TA Bíscaro and S Kashima (First Brazilian Symposium of Basic Research in HIV/ AIDS 1995) to evaluate HIV-1 positive samples from São Paulo, showing an unusually high prevalence (79\%) of those containing the GW motif.

We present here a rapid screening for the typical Brazilian HIV-1 subtype B variant B" based on the association of HMA using sets of primers able to amplify the $\mathrm{C} 2-\mathrm{C} 3$ region (640bp), with a further digestion step of the corresponding PCR product using the Fok I restriction enzyme, in order to evaluate the frequency of these viruses and its potential association with modes of transmission and exposure categories of HIV-1 infected individuals from Rio de Janeiro.

Briefly, HIV-1 proviral DNA samples were obtained from peripheral blood mononuclear cells of HIV-1 seropositive individuals and AIDS patients participating in distinct research protocols from 1990 to 1996 as follows: 30 men enrolled in the cohort of heterosexual transmission of HIV in Rio de Janeiro, 93 outpatients followed at two medical centers in Rio de Janeiro (Hospital Evandro Chagas, Instituto Oswaldo Cruz and Ambulatório do Banco da Providência) and, 20 injecting drug users (IDUs) recruited both from treatment centers and the streets, as part of an ongoing cooperative multicenter project (Projeto Brasil). For subtype determination, proviral DNA samples were previously PCR amplified by a nested protocol using sets of primers which amplify a $2.0 \mathrm{~Kb}$ fragment containing the envelope region in the first round (primers ED3/ED14), and a 600bp fragment of the $\mathrm{C} 2-\mathrm{C} 3$ envelope region for the 2 nd round PCR (primers ED31/33). PCR conditions and HMA subtyping were performed as described elsewhere (Delwart et al. loc. cit., Bachmann et al. loc. cit.). Only HIV-1 subtype B samples were selected for this study. After subtype determination, $10 \mathrm{ml}$ of the amplified DNA were digested with 6U Fok I restriction enzyme, in a final vol- 
ume of $25 \mathrm{ml}$ of $1 \mathrm{x}$ buffer supplied by the manufacturer (Amersham Life Science, Ill, USA), for 2 $\mathrm{hr}$ at $37^{\circ} \mathrm{C}$. DNA samples were submitted to electrophoresis through $2 \%$ agarose gels for $1 \mathrm{hr}$ at $50 \mathrm{~V}$ in $1 \mathrm{xTBE}$ (45mMTris-borate, $1 \mathrm{mM}$ EDTA) and the restriction fragments were evaluated under UV illumination. The molecular weight of the restriction fragments was determined based on the migration of the phiX- HaeIII digested molecular weight marker (Sigma, Chem. Co., Mo, USA).

The digestion of the 640bp PCR amplified products with Fok I restriction enzyme showed two major patterns, one non digested (640bp), corresponding to the North American/European subtype B samples, and a second one with two fragments of $410 \mathrm{bp}$ and $230 \mathrm{bp}$, typical for the GWGR Brazilian subtype B samples. These results agree with the expected size of the fragments based on the restriction map of HIV-1 subtype B and B" variant samples available in the Genebank. Moreover, three other patterns were also observed in a lower frequency, showing additional Fok $I$ restriction sites, as showed in Figure. DNA sequencing of these samples was performed in order to confirm the presence of these Fok I restriction sites outside the V3 loop (data not shown).

Using this approach $59(41 \%)$ out of the 143 subtype B samples from Rio de Janeiro included in this study were identified as corresponding to the typical GWGR subtype B isolates. Fifty-six (95\%) showed two Fok I restriction fragments of $410 \mathrm{bp}$ and $230 \mathrm{bp}$, whereas $3(5 \%)$ showed three restriction fragments of $410 \mathrm{bp}, 120 \mathrm{bp}$ and $80 \mathrm{bp}$. For the remaining 84 subtype B samples, 77 (92\%) showed the undigested pattern, while $6(7 \%)$ showed two Fok I restriction fragments of 500bp and 140bp and 1 sample showed two restriction fragments of $350 \mathrm{bp}$ and $290 \mathrm{bp}$. The presence of the $410 \mathrm{bp}$ or $230 \mathrm{bp}$ Fok I fragments seems to be discriminative of the B" Brazilian HIV-1 samples and attention has to be payed as even conventional B subtype isolates can be digested with this restriction enzyme resulting in closer patterns.

As shown in Table, similar proportions of B and B" isolates were detected independently of the exposure category of the infected individuals. No association could be verified with the distribution of the B" variant (Fisher's exact test) when patients were classified by gender $(\mathrm{p}=0.855)$ or by sexual and parenteral routes of transmission $(\mathrm{p}=0.657)$.

These data suggest that the B" variant probably emerged or was introduced since the beginning of AIDS epidemic in Brazil and is spread among all exposure categories, as the other subtype B samples. Indeed, one of the patients was

\section{TABLE}

Distribution of the typical HIV-1 Brazilian subtype B variant (B") by exposure categories of infected individuals in Rio de Janeiro City, Brazil

\begin{tabular}{lrrrr}
\hline Categories & \multicolumn{3}{c}{ HIV-1 subtype B } & Total \\
\cline { 2 - 3 } & \multicolumn{1}{c}{ B" ${ }^{a}(\%)$} & \multicolumn{1}{c}{ B $(\%)$} & \\
\hline Homosexual/ & $24(43 \%)$ & $32(57 \%)$ & $56(100)$ \\
Bisexual & & & & \\
Heterosexual & $23(41 \%)$ & $33(59 \%)$ & $56(100)$ \\
IDUs $b$ & $6(30 \%)$ & $14(70 \%)$ & $20(100)$ \\
Transfusion & $3(60 \%)$ & 2 & $(40 \%)$ & $5(100)$ \\
ND & $3(50 \%)$ & $3(50 \%)$ & $6(100)$ \\
\hline Total & $59(41 \%)$ & $84(59 \%)$ & $143(100)$ \\
\hline
\end{tabular}

$a$ : determined by the Fok I restriction fragment pattern; ND not determined.

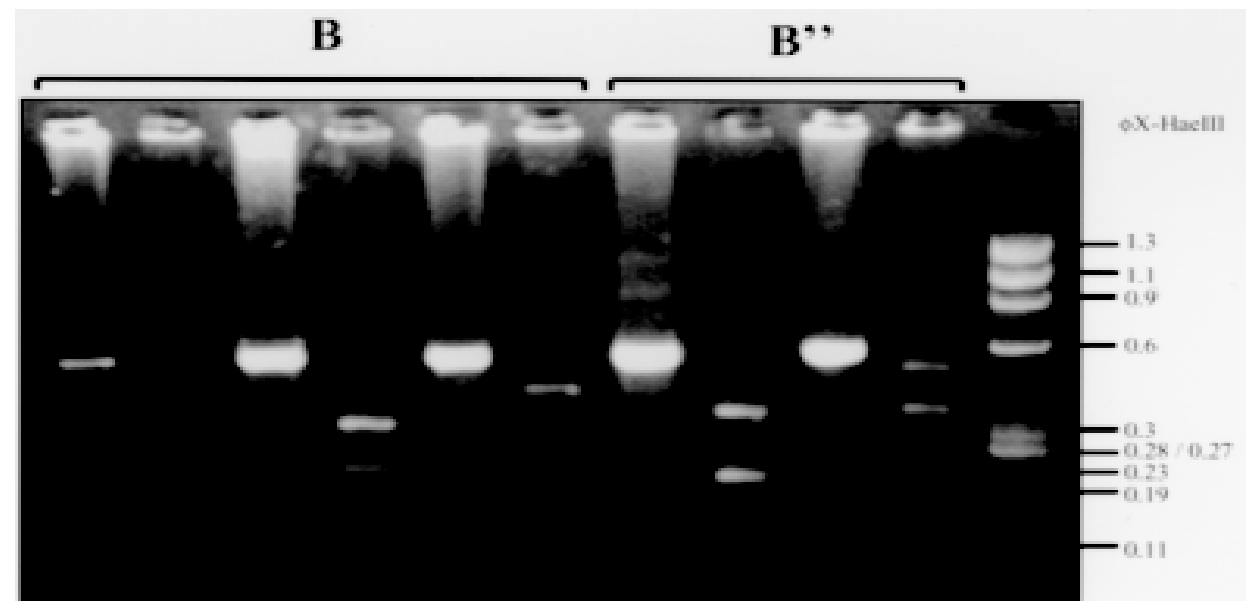

Fok I restriction fragment length polymorphism of Brazilian subtype B samples. PCR amplified HIV-1 envelope fragments spanning the C2-C3 region digested with Fok I restriction enzyme and electrophoresed in 2.4\% agarose gel. Phi-X HaeIII was used as molecular weight marker. 
infected with HIV-1 B" variant during a blood transfusion after giving birth in 1996, and another case (IDU) has a positive serology for HIV since 1987. Moreover, the presence of the GWGR variant was also verified in sera collected in 1983 in São Paulo (Hendry et al. 1996 loc. cit.). The proportion of $40 \%$ of the B" isolates among B subtype samples seems to be maintained over time, as similar results have been verified in previous studies comparing HIV-1 samples obtained at different time points (Potts et al. 1993 loc. cit., Morgado et al. 1994 loc. cit.).

The importance of this subtype B variant in the AIDS epidemic in Brazil is not well understood.
We do not know whether the genetic and antigenic polymorphism verified in the gp120 V3 loop of these samples is also extended to other regions of the virus genome, mainly those encoding antigenically relevant epitopes for neutralizing antibodies and cell mediated immunity. Extensive cross neutralization were observed between B and B" isolates suggesting the conservation of neutralizing epitopes among these samples outside the V3 loop (V Bongertz et al. 1998 Scand J Immunol 47: in press). Further studies will be necessary to evaluate the extent of the diversity of B" variants as well as its potential implications on vaccine design and pathogenesis. 